\title{
Learning with prolonged delay of reinforcement'
}

\author{
JOHN GARCIA, FRANK R. ERVIN² AND ROBERT A. KOELLING \\ DEPARTMENT OF PSYCHIATRY AND NEUROLOGY, \\ HARVARD MEDICAL SCHOOL AND NEUROSURGICAL SERVICE, MASSACHUSETTS GENERAL HOSPITAL
}

Gustatory aucrsions, induced in rats by conditionally pairing a distinctive flavor with a noxious drug, were readily established even when injections were delayed an hour or more. The optimal interstimulus interval and effectiveness of iues for leaming appear to be a function of the specific effects of the reinforcer on the organism.

It is considered axiomatic in theory and practice that no learning will occur without immediate reinforcement. For example, a hungry rat will not learn to press a lever for food unless the response is immediately followed by food (primary reinforcement) or by a signal which has been associated with food in the past (secondary reinforcement). Food can be described as rewarding, but the same general rule has been applied to punishing agents also. Delays of the order of 3 to 45 sec. have a deleterious effect upon learning in a wide variety of experimental situations. The significance of these findings for reinforcement theory was discussed by Spence (1947) and a recent review (Renner, 1964) reveals there has been no major modification of the temporal contiguity aspect. However, our data indicates that immediate reinforcement is not a general requirement for all learning.

Method

Young adult male rats (Sprague-Dawley, 300 to $400 \mathrm{gm}$ ) were maintained in individual cages with Purina Laboratory Chow ad lib. Drinking was restricted to a 10-min. period each day. After one week of habituation to this schedule, treatment began.

In Experiment $A$, five groups $(\mathrm{N}=8$ each) were treated. One experimental group (Sac-Apo:inj) was given a gustatory cue in its drinking water ( $1 \mathrm{gm}$ saccharine per liter) and after a delay was injected with a drug which produced gastric disturbances $(7 \mathrm{mg} / \mathrm{kg}$ apomorphine hydrochloride I.P.). The animals were injected in serial order at $1-\mathrm{min}$. intervals with the first animal injected at $5 \mathrm{~min}$. and the last one at $12 \mathrm{~min}$. after the saccharin water bottle was removed from the home cage. One control group (Sac-Sal:inj) drank saccharinwater and was injected with saline, while another control (Wat-Apo:inj) drank water and was injected with apomorphine. An additional experimental group (SacApo:inj) received delayed injections in serial order from 15 to $22 \mathrm{~min}$. post-drinking. Other rats (Sac-Shock), immediately after drinking saccharin-water, were taken from their cages and placed in a box with an electric grid floor and three shocks $(0.5 \mathrm{sec}$. pulses at $3 \mathrm{ma})$ were delivered within $1 \mathrm{~min}$. to the paws.

All groups received four treatments, one every third day and then three extinction tests (i.e. no injections or shock) on the same schedule. Between treatment days, the animals were given water for $10 \mathrm{~min}$.
In Experiment $\mathrm{B}$, five experimental groups ( $\mathrm{N}=6 \mathrm{each})$ drank saccharin-water and received apomorphine injections (15mg/kg I.P.) with delays of $30,45,75,120$ and $180 \mathrm{~min}$. Five treatments were administered, one every third day. One control group drank saccharin-water but received no injection.

\section{Results and Discussion}

The apomorphine injected animals (Sac-Apo:inj) displayed a progressive decrease in intake of saccharinwater indicating that the pairing of this distinctly flavored fluid with the drug effects produced a gustatory aversion (Fig. 1). The difference in saccharin-water intake between the apomorphine injected group and their saline injected controls (Sac-Sal:inj) was statistically significant after two injections ( $p<.01$ by ranks test). The decrement increased following each drug administration and then was reversed during the extinction trials. Apomorphine injections had no effect on those animals (Wat-Apo:inj) which drank water every day, demonstrating that differential reinforcement of the gustatory cue is necessary to produce the fluid intake decrement (Fig. 1).

The magnitude of the decrement in saccharin intake produced by four doses of apomorphine (Sac-Apo:inj) was independent of the delay of the reinforcing injection $(\mathrm{r}=0)$ from 5 to $22 \mathrm{~min}$. Furthermore, Experiment B indicated that five apomorphine injections could produce a significant effect at delays up to $75 \mathrm{~min}$. (Fig. 2).

Apomorphine causes nausea and emesis in humans, but in rats emesis is blocked by the cardiac sphincter. Our doses caused the animals to stop eating for $30 \mathrm{~min}$. and caused visible signs of illness within several minutes in most animals.

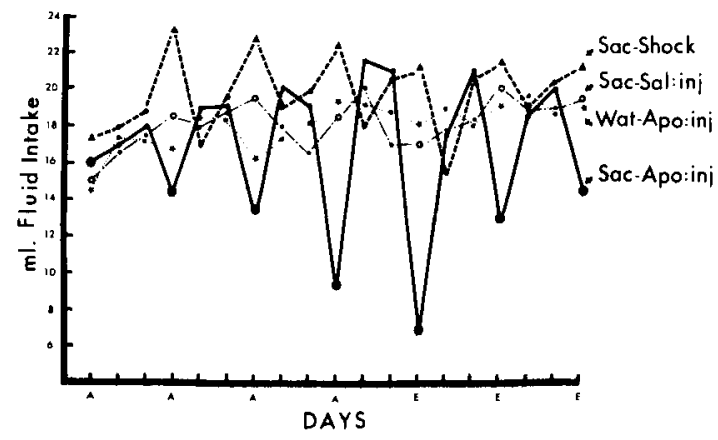

Fig. 1. Experiment A: Daily mean fluid intake during a tenminute period on acquisition days ( $A$ ) extinction days $(E)$ and water intake on days between treatments. Only the group receiving Saccharin followed by an apomorphine injection (Sac:Apo:inj) learned to reduce saccharin-water intake. Neither apomorphine (Wat-Apo:inj) nor saccharin alone (Sac-Sal:inj) had an appreciable effect, while Saccharin followed by shock (Sac-Shock) had a converse effect. 


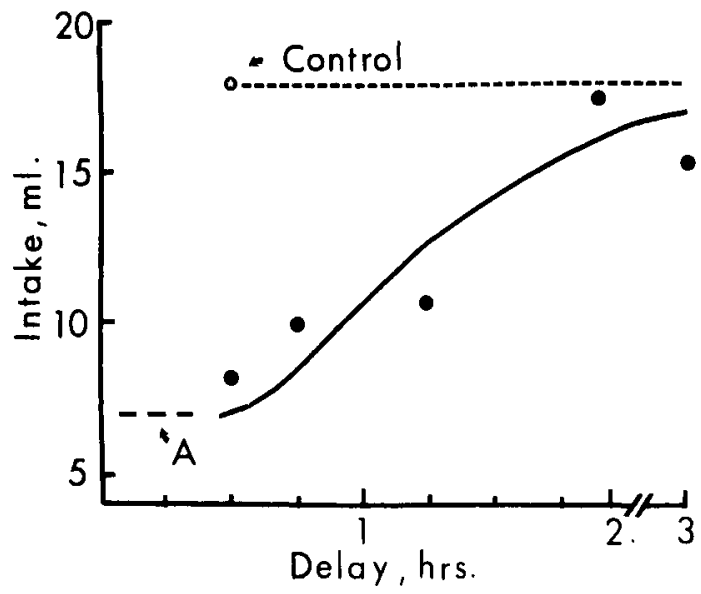

Fig. 2. Experiment B: Mean fluid intake of saccharin-flavored water on the first extinction day, following five conditional pairings of saccharin and apomorphine for groups differing in delay of injection. Results of Experiment $A$ are also indicated.

The rats receiving electrocutaneous shock (Sac-Shock) displayed a significant increase in saccharin-water intake after the first shock (Fig. 1). This increase may be due to the activating effects of shock rather than to associative learning. However, the contrast between shock rats and drug rats in their responses to handling and treatment was also marked. The drug and control animals progressively habituated to handling and injection, but the shock animals made strong attempts to escape after the first shock trial. This observation supports a previous study indicating that the avoidance reactions induced by electrocutaneous shock were readily transferred to auditory and visual signals but not to gustatory ones. Conversely, the avoidance reactions induced by $\mathrm{X}$-ray or toxin were readily transferred to gustatory cues but not to auditory and visual ones (Garcia \& Koelling, 1966).

Two sources, outside of the traditional laboratory studies of learning, indicate similar learning with prolonged delays of reinforcement. Aversions have been produced with a discrimination learning paradigm where one gustatory cue is paired with ionizing radiation and an alternative cue is not so paired (Garcia et al, 1961). Yet, this effect of radiation is not manifested until over an hour post-exposure (Smith et al, 1965). Aversions have been established for unflavored water and for merely sniffing a distinctive odor during exposure, indicating that the phenomenon is not dependent upon lingering traces of a strongly flavored solution (Garcia \& Koelling, 1965). Furthermore, ani- mals on water-deprivation invariably consume a large meal of dry food so that the gustatory and olfactory cues are immediately followed by intervening stimulation via these modalities which is not differentially reinforced.

Rats utilizing olfaction and gustation also learn to avoid poisons with slow cumulative effects, such as dicoumarin which gradually reduces the clotting power of the blood and eventually causes internal bleeding. These bait-shy responses in many cases do not develop until hours after the ingestion of the bait (Barnett, 1963).

These data indicate anew that the mammalian learning mechanisms do not operate randomly, associating stimuli and reinforcers only as a function of recency, frequency and intensity. The omnivorous rat displays a bias, probably established by natural selection, to associate gustatory and olfactory cues with internal malaise even when these stimuli are separated by long time periods. Auditory, visual and tactual stimuli are not so readily associated with malaise, though they also are discriminable and informative, i.e. suprathreshold and differentially reinforced. On the other hand, the latter cues are more readily associated with peripheral pain. In this case, both cue and reinforcer are localized in external space by the rat and under "natural" conditions, escape movements are particularly adaptive. This bias has a practical diagnostic value for drug research. Since the gustatory aversion is sensitive to toxicity yet insensitive to peripheral pain, it can be conveniently used in conjunction with injection and surgical procedures.

\section{References}

Bamett, S. A. The rat: A study in behavior. Chicago: Aldine Press, 1963.

Garcia, J., Kimeldorf, D. J., \& Hunt, E. L. The use of ionizing radiation as a motivating stimulus. Psychol. Rer., 1961, 68, 383-395.

Garcia, J., \& Koelling, R. A. A comparison of aversions induced by $\mathrm{X}$-rays, toxins and drugs in the rat. Radiat. Res., 1965, in press.

Garcia, J., \& Koelling, R. A. Relation of cue to consequence in avoidance learning. Psychon. Sci., 1966, 4, 123-124.

Renner, K. E. Delay of reinforcement: A historical review, Psychol. Bull., 1964, 5, 341-361.

Spence, $\mathbf{K}$. W. The role of secondary reinforcement in delayed reward learning. Psychol. Rev., 1947, 54, 1-8.

Smith, J. C., Tay lor, H. L., Morris, D. D., \& Hendricks, J. Further studies of $\mathrm{x}$-ray conditioned aversion during the post exposure period. Radiat. Res., 1965, 24, 423-431.

\section{Notes}

1. This research was supported by Grant No. CA-07368 and NASA Contract No. NsG262-63.

2. NIH Career Award No. K3-MH-19.434. 